\title{
Self-cloning CRISPR/Cpf1 facilitated genome editing in Saccharomyces cerevisiae
}

\author{
Zhen-Hai Li, Feng-Qing Wang* and Dong-Zhi Wei*
}

\begin{abstract}
Background: Saccharomyces cerevisiae is one of the most important industrial microorganisms. A robust genome editing tool is vital for both fundamental research and applications. To save the time and labor consumed in the procedure of genome editing, a self-cloning CRISPR/Cpf1 system (sCCRISPR/Cpf1), in which a self-cleaving plasmid and PCR-generated site-specific crRNA fragment were included, was developed.

Results: Using scCRISPR/Cpf1 as the genetic tool, simple and fast singleplex and multiplex genomic integration of in vivo assembled DNA parts were investigated. Moreover, we validate the applicability of scCRISPR/Cpf1 for cell factory development by creating a patchoulol production strain through two rounds of iterative genomic integration. The results showed that scCRISPR/Cpf1 enables singleplex and tripleplex genomic integration of in vivo assembled DNA parts with efficiencies of 80 and 32\%, respectively. Furthermore, the patchoulol production strain was successfully and rapidly engineered and optimized through two rounds of iterative genomic integration by scCRISPR/Cpf1.

Conclusions: scCRISPR/Cpf1 allows for CRISPR/Cpf1-facilitated genome editing by circumventing the step to clone a site-specific crRNA plasmid without compromising efficiency in S. cerevisiae. This method enriches the current set of tools available for strain engineering in S. cerevisiae.
\end{abstract}

Keywords: CRISPR/Cpf1, Genome editing, Self-cloning, Saccharomyces cerevisiae

\section{Background}

In recent years, Saccharomyces cerevisiae has served as an important platform organism for bio-based production of an ever-increasing list of biofuels, bulk chemicals, and pharmaceuticals in a sustainable and green way (Engels et al. 2008; Kondo et al. 2012; Li et al. 2016; Marienhagen and Bott 2013). Until recently, various important molecular compounds, such as artemisinin precursor, farnesene, and ginsenosides, have been synthesized with high efficiency in S. cerevisiae facilitating the rapid evolution of the fields of metabolic engineering and synthetic biology (Dai et al. 2014; Ro et al. 2006; Meadows et al. 2016; Yu et al. 2017). The design-build-test strategy of synthetic biology involves the construction and optimization of cell factories, often requiring dramatic reconstruction and frequent debugging of metabolic networks of S. cerevisiae

\footnotetext{
*Correspondence: fqwang@ecust.edu.cn; dzhwei@ecust.edu.cn State Key Lab of Bioreactor Engineering, Newworld Institute of Biotechnology, East China University of Science and Technology, Shanghai 200237, China
}

(Carbonell et al. 2016; Nielsen and Keasling 2016; Paddon and Keasling 2014; Smanski et al. 2014). Efficient methods for genetic manipulation are required for balanced multi-step metabolic pathway integration to investigate optimal combinations for the synthesis of target products in S. cerevisiae. The efficient homologous recombination (HR) machinery of S. cerevisiae has allowed the integration of DNA molecules into chromosome with appreciable efficiency. Although the genomic integration of DNA parts facilitated by selection markers is well-developed in S. cerevisiae, iterative genome editing remains timeand labor-consuming due to marker recycling (Siddiqui et al. 2015; Xie et al. 2013). The native HR machinery of S. cerevisiae is not efficient enough for the complex and marker-free gene targeting required for modern synthetic biology (Storici et al. 2001). However, the efficiency of genome editing mediated by HR can be dramatically enhanced when a double-stranded break (DSB) is introduced into the genome during transformation (Storici et al. 2003). With the booming of Clustered Regularly Interspaced Short Palindromic Repeats (CRISPR)-Cas9, 
which allows for efficient generation of DSBs in chromosomes, marker-free approaches have thus emerged as the preferred scheme in genome editing.

The CRISPR/Cas9 system from Streptococcus pyogenes has already been widely used in various organisms (Hwang et al. 2013; Sander and Joung 2014; Wang et al. 2017; Westbrook et al. 2016). The possibility of applying the CRISPR/Cas9 system to more complex engineering tasks can be raised by native HR (Schwartz et al. 2017; Yao et al. 2017). Due to the industrial importance of S. cerevisiae, it is of high priority to find a way to take advantage of the highly efficient CRISPR/Cas9 system for robust genome editing. Numerous reports have described the metabolic engineering of $S$. cerevisiae using the CRISPR/Cas9 system. In 2013, Dicarlo et al. (2013) first reported that DSB could be effectively generated in the yeast genome using the CRISPR/ Cas9 system. Jakočiūnas et al. (2015) have reported a CasEMBLR method for marker-free multiloci integration of in vivo assembled DNA parts. Horwitz et al. (2015) have shown that multiplexed integration of large constructs could be carried out. Zhou et al. (2016) developed a CRISPR/Cas9-based CasHRA method for sequential genome editing. The methods mentioned above have greatly enhanced the efficiency of genome editing in S. cerevisiae. Nevertheless, construction of multiplexed-gRNA plasmid is time- and labor-consuming in iterative genome editing.

Cpf1, a newly identified family of class 2/type $\mathrm{V}$ CRISPR bacterial endonucleases, shows some distinct features compared to Cas9, such as: (i) Cpf1 is guided only by a single crRNA and displays an activity of crRNA processing, which may simplify multiplex genome editing; (ii) the Protospacer Adjacent Motif (PAM) of Cpf1 is T-rich, which is located at the $5^{\prime}$ end of the protospacer (Zetsche et al. 2017). The distinctive features could facilitate Cpf1 as an attractive and alternative CRISPR-Cas system for genome editing. Although, Cpf1 has been recently reported on efficient genome editing in S. cerevisiae, more research should be done to extend its application (Swiat et al. 2017; Verwaal et al. 2018).

Arbab et al. (2015) have reported a self-cloning CRISPR/Cas9 system (scCRISPR/Cas9) for CRISPR/ Cas9-mediated genomic mutation and site-specific knockin transgene creation in mouse and human embryonic stem cells as well as HEK293T cells. The critical parameter for scCRISPR/Cas9 is the self-cleaving of the sgRNA plasmid, which contains a self-complementary palindromic CRISPR (scCRISPR), and the following plasmid repair by HR.

In this study, we present a CRISPR/Cpf1-based method that allows for simple and fast genome editing $(1 \mathrm{~h}$ for crRNA preparation from primer arrival versus 2 days for conventional gRNA plasmid construction) by circumventing any cloning steps in the genome editing process. This method enables singleplex and tripleplex genomic integration of in vivo assembled DNA parts with efficiencies of 80 and $32 \%$, respectively. Using this method, $S$. cerevisiae was successfully and rapidly optimized for the production of patchoulol by over-expressing three genes, replacing one promoter, and blocking two genes through two rounds of iterative genomic integration.

\section{Results}

\section{Rationale and design of scCRISPR/Cpf 1}

Considering that the crRNAs for Cpf1 can be expressed in a tandom crRNA array, yeast cells are known to promote reconstruction of a linearized plasmid DNA through homologous recombination (plasmid HR), and the HR pathway could be stimulated by DSB, a selfcloning CRISPR/Cpf1 system (scCRISPR/Cpf1) that was developed in this study to allow for CRISPR/Cpf1-mediated genome editing in S. cerevisiae.

scCRISPR/Cpf1 relies on the yeast cells to clone the desired crRNA plasmid in vivo. Therefore, we constructed a plasmid co-expressing a self-complementary palindromic crRNA and Cpf1 (pSC-Pal) (Fig. 1a). Upon transcription and translation in yeast cells, the complex of Cpf1 and palindromic crRNA should generate DSB in the palindromic crRNA region of pSC-Pal (Fig. 1b). The DSB could be reconstructed with a small DNA fragment containing a desired site-specific crRNA into a functional site-specific crRNA and Cpf1 co-expression plasmid by plasmid HR (Fig. 1a, b). The whole process above was carried out in the yeast cell, and the small DNA fragment containing a desired site-specific crRNA array can be generated rapidly by PCR within $1 \mathrm{~h}$ once the primers were acquired. Furthermore, pSC-Pal derivatives with the URA3 marker inside yeast cells could be eliminated easily by counterselection on plate containing 5 -fluoroorotic acid, which facilitates the cells to be ready for next round genome editing (Xie et al. 2015).

\section{SCCRISPR/Cpf1 for genome editing S. cerevisiae}

To test whether scCRISPR/Cpf1 functions to self-cleave in $S$. cerevisiae, the URA3 plasmid co-expressing Cpf1 and non-palindromic crRNA (pCon-NP) and the URA3 plasmid co-expressing Cpfland palindromic crRNA but missing the corresponding PAM sequence (pCon-MP) were constructed (Additional file 1: Table S1). The yeast cells transformed with pSC-Pal, pCon-NP, and pCon-MP, respectively, were plated on SD-URA plates, respectively. The results showed that there were many transformants on both plates of pCon-NP and plates of pCon-MP; while no transformants were found on plates of $\mathrm{pSC}$ Pal (Fig. 2a). The results demonstrated that the pSC-Pal 
$\mathbf{a}$

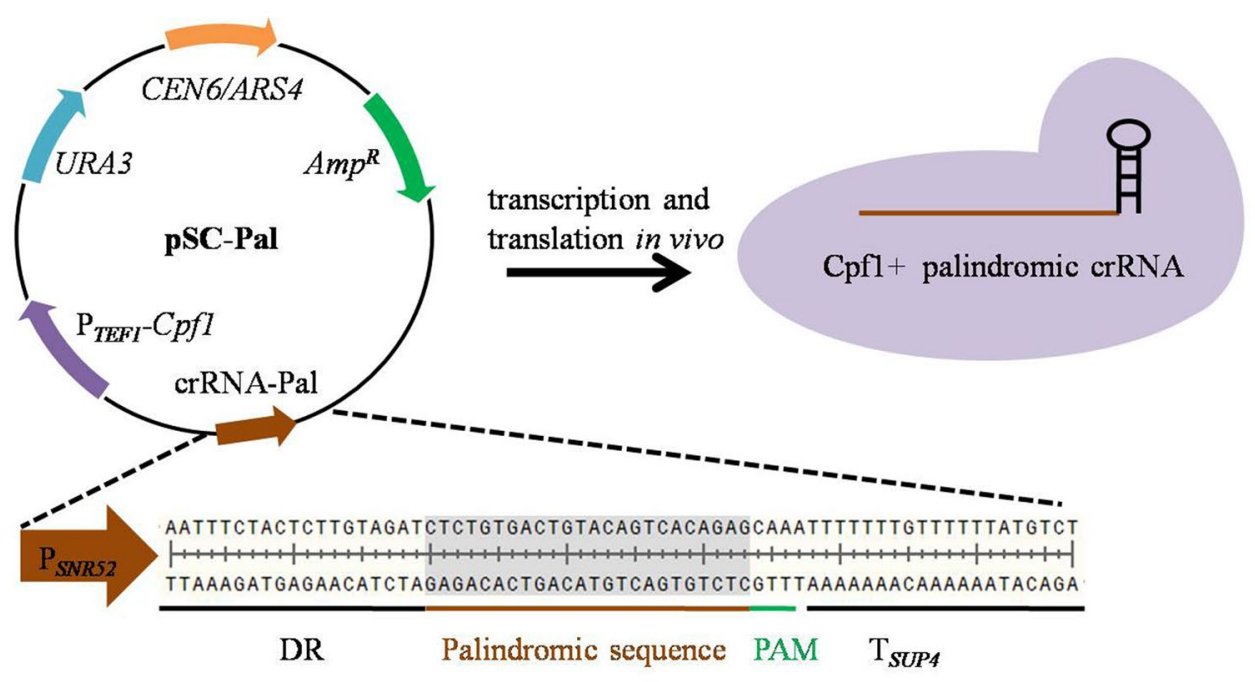

b

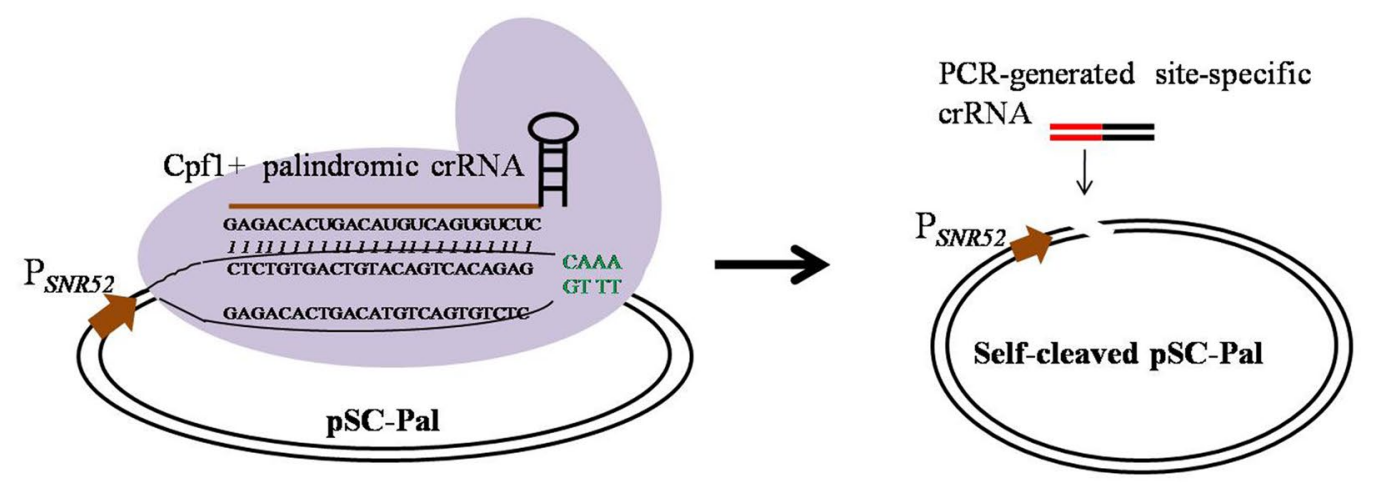

Cpf1-Palindromic crRNA complex cleaves complementary pSC-Pal

cleaved pSC-Pal is repaired by plasmid HR with a PCR-generated site-specific crRNA homology template

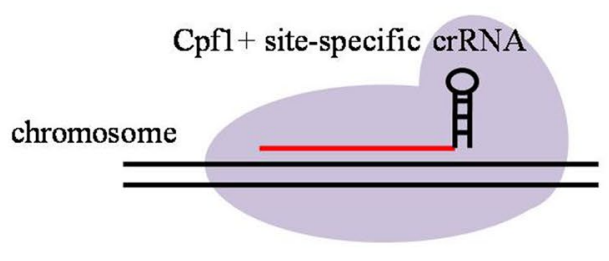

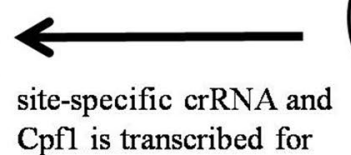
Cpfl is transcribed for genomic targeting $\downarrow$ site-specific crRNA

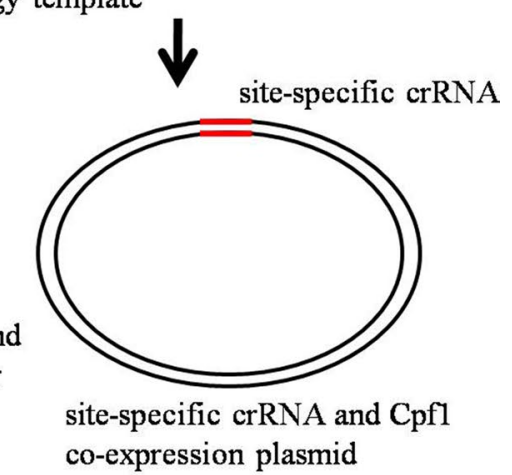

Fig. 1 Schematic of sCCRISPR/Cpf1. a pSC-Pal: the self-cleaving complementary palindromic crRNA and Cpf1 co-expression plasmid. b Schematic shows the scCRISPR/Cpf1 process that occurs inside target yeast cells. CEN6/ARS4, replication origin; URA3, selection marker used in yeast; $A m p^{R}$, selection marker used in E. coli; $\mathrm{P}_{\text {TEF } 1}-\mathrm{Cpf1}$, the $\mathrm{Cpf1}$ expression cassette driven by promoter $\mathrm{P}_{\text {TEF } 1}$; $\mathrm{DR}, 19$ bp direct repeat which shown in black; PAM, protospacer-associated motif sequence; $P_{\text {SNR522 }}$ SNR52 promoter; $T_{\text {SUP4 }}$ SUP4 terminator; the complementary palindromic sequence of crRNA was shown in brown and the site-specific sequence of crRNA was shown in red 


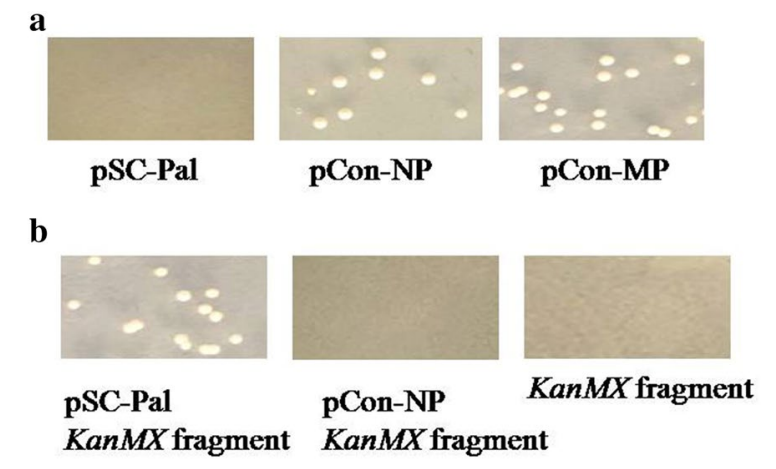

PAM the $A D E 2$ target sequence

TITCCCGGTTGTGGTATATTTGTGTG

d

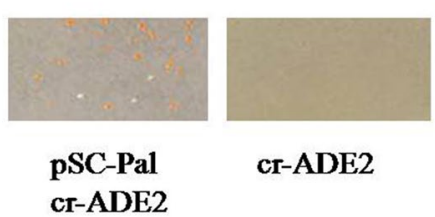

Fig. 2 Functionality of scCRISPR/Cpf1. a Pictures of transformants obtained by transforming pSC-Pal (left), pCon-NP (middle) or pCon-MP (right). b Pictures of transformants obtained by transforming the KanMX fragment with pSC-Pal (left), the KanMX fragment with pCon-NP (middle) or the KanMX fragment only (right). c The cr-ADE2 target in the ADE2. d Pictures of transformants obtained by transforming cr-ADE with pSC-Pal (left) or cr-ADE only (right)

was self-cleaved and the self-cleaved linearized pSC-Pal was lost without repair. Thus, scCRISPR/Cpf1 functions to self-cleave in $S$. cerevisiae. Furthermore, the presence of both PAM sequence and the self-complementary palindromic crRNA in the plasmid is essential for the selfcleaving of pSC-Pal.

To test whether plasmid HR of yeast functions to repair self-cleaved linearized pSC-Pal in S. cerevisiae, the long fragment was first used. The $\operatorname{KanMX}$ fragment flanked with $90 \mathrm{bp}$ that overlaps with each end of linearized pSCPal was amplified from the plasmid pUMRI-15. The yeast cells transformed with the $\operatorname{KanMX}$ fragment alone, the $K a n M X$ fragment with pCon-NP, and the KanMX fragment with pSC-Pal, respectively, were plated on SD-URA plates containing the geneticin G418, respectively. The results showed that there were no transformants on both plates of the $K a n M X$ fragment alone and plates of the KanMX fragment with pCon-NP; while many transformants were found on plates of the $\operatorname{KanMX}$ fragment with pSC-Pal (Fig. 2b). The results demonstrated that the selfcleaved linearized pSC-Pal was repaired with the KanMX fragment by plasmid HR in S. cerevisiae. To test whether plasmid $\mathrm{HR}$ of yeast functions to repair self-cleaved linearized pSC-Pal with a small site-specific crRNA fragment flanked with $90 \mathrm{bp}$ that overlaps with each end of linearized pSC-Pal in S. cerevisiae, a small site-specific crRNA fragment targeting the $A D E 2$ (cr-ADE2) was generated by PCR (Fig. 2c). The mutagenesis of the $A D E 2$ gives the yeast mutants a visible red phenotype. To eliminate the disturbance of the URA3 in pSC-Pal, the $U R A 3$ in $\mathrm{pSC}$-Pal was replaced with the LEU2, generating the plasmid pLEU-Pal. The yeast cells transformed with the cr-ADE2 alone and the cr-ADE2 with pLEU-Pal, respectively, were plated on SD-LEU plates, respectively. The results showed that there were no transformants on plates of the cr-ADE2 alone, while $90 \%$ of red transformants were found on plates of the cr-ADE2 with pLEU-Pal (Fig. 2d). The results demonstrated that the self-cleaved linearized pLEU-Pal was repaired with the cr-ADE2 fragment by plasmid HR in S. cerevisiae. Thus, scCRISPR/Cpf1 functions to generate genomic mutation with small site-specific crRNA fragment in $S$. cerevisiae.

To test whether scCRISPR/Cpf1 functions to facilitate singleplex genomic integration of in vivo assembled DNA parts in S. cerevisiae, a crRNA targeting the Gal1-7 locus, cr-Gal17, was randomly selected and constructed (Additional file 1: Fig. S1A). The $\alpha$-galactosidase encoding gene $M E L 1$ from S. cerevisiae Y187 was designed to replace the Gal1-7 in the genome of S. cerevisiae BY4741 (Fig. 3a). The substrate of X- $\alpha$-gal could be catalyzed by the MEL1 into a visible blue product, which facilitated phenotypic analysis. Up to $80 \%$ of blue transformants were obtained through co-transformation of the pSC-Pal, the corresponding donor DNA fragments of the $M E L$, and cr-Gal17, whereas no any blue transformant was obtained through transformation of the corresponding donor DNA with cr-Gal17, the corresponding donor DNA with pSC-Pal, and the corresponding donor DNA alone, respectively (Fig. 3b, c). Correct integration of the MEL1 fragment at the Gal1-7 locus was verified by PCR using primer pair SQ1/SQ2, and we confirmed that all the 6 blue colonies randomly selected had a correct integration profile (Fig. 3a, d). To assess accuracy of plasmid HR in the blue transformants, the crRNA cassette of 6 blue colonies randomly selected was amplified and sequencing with primer pair $\mathrm{cr}-\mathrm{F} / \mathrm{cr}-\mathrm{R}$ was performed. The sequencing results showed that the pSCPal was successfully recombined into a crRNA plasmid expressing cr-Gal17 in each of the 6 blue transformants. Hence, scCRISPR/Cpf1 performs efficient and faithful crRNA recombination and allows for efficient singleplex genomic integration of in vivo assembled DNA parts in S. cerevisiae.

The rate-limiting step of the mevalonate (MVA) pathway could be released by over-expressing the truncated 3-hydroxy-3-methylglutaryl-coenzyme-A reductase 
a

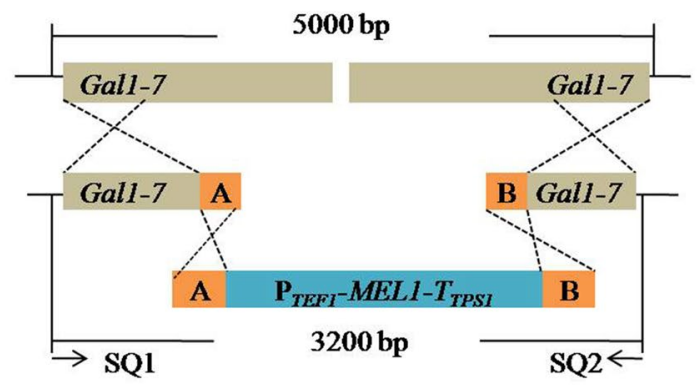

c

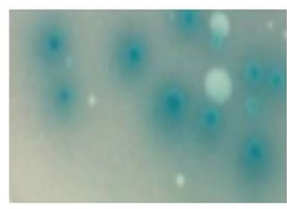

pSC-Pal

donor DNA b

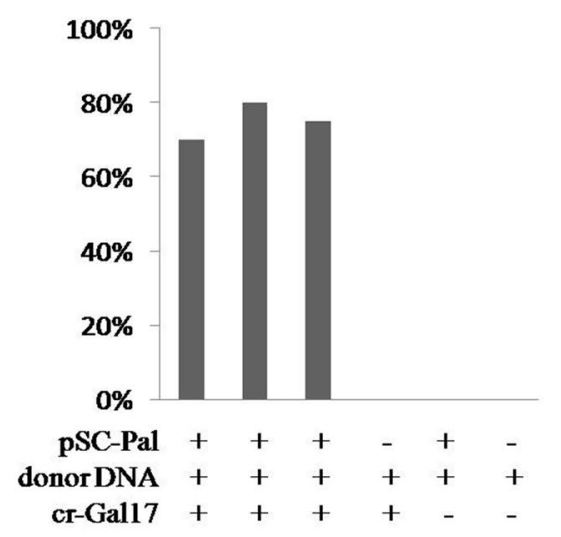

d

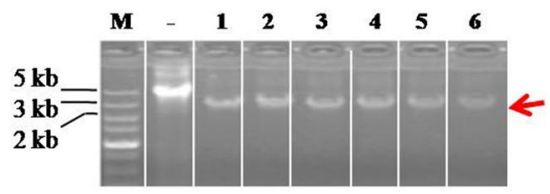

Fig. 3 scCRISPR/Cpf1 facilitated singleplex genomic integration of in vivo assembled DNA parts in S. cerevisiae. a sCCRISPR/Cpf1 facilitated singleplex genomic integration of the MEL 1 into the Gal1-7. b Efficiency of singleplex genomic integration assisted by scCRISPR/Cpf1. c Pictures of transformants obtained by co-transforming donor DNA of MEL 1 with pSC-Pal and Cr-Gal17. d PCR result of 1 white transformant (-) and 6 blue transformants, lines \#1-6, using primer pair SQ1/SQ2. a, b in orange rectangle: 50 bp homologous connector sequences. $P_{\text {TEF1, }}$ Promoter of the TEF1;

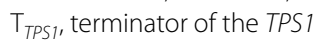

(tHMG1) in S. cerevisiae (Xie et al. 2015). Co-expression of the $t H M G 1$ and the three genes of $\operatorname{crtE}$, crtYB, and $\operatorname{crtI}$ from Xanthophyllomyces dendrorhous enable the efficient production of $\beta$-Carotene, which gives $S$. cerevisiae a visible orange phenotype (Xie et al. 2015).

To test whether scCRISPR/Cpf1 functions to facilitate tripleplex genomic integration of in vivo assembled DNA parts in $S$. cerevisiae, a DNA fragment containing a crRNA array sequence targeting the Gal1-7, the Gal80, and the HO locus flanked with 50 bp that overlaps with each end of self-cleaved linearized pSC-Pal (cr-Gal17/Gal80/HO) was constructed for plasmid $\mathrm{HR}$, and the individual $c r t$, $\operatorname{crt} Y B$, and $t H M G 1-c r t E$ expression cassettes were used to replace the Gal17, the Gal80, and the $H O$, respectively (Fig. 4a, Additional file 1: Fig. S1A). Up to $32 \%(480 / 1500)$ of orange transformants were obtained through the co-transformation of the cr-Gal17/Gal80/HO, the corresponding donor DNA, and pSC-Pal, whereas no orange transformant was obtained through transformation of the corresponding donor DNA with the cr-Gal17/Gal80/ $\mathrm{HO}$, the corresponding donor DNA with pSC-Pal, and the corresponding donor DNA alone, respectively
(Fig. 4b, c). Correct integration of the $\beta$-carotene synthetic pathway was verified by PCR using primer pairs SQ1/SQ2, SQ3/SQ4, and SQ5/SQ6, respectively, and we confirmed that all the 6 orange colonies randomly selected had a correct integration profile (Fig. 4a, d). HPLC results showed that $\beta$-carotene was synthesized in all the three orange strains randomly selected, demonstrating that the $\beta$-carotene synthetic pathway was successfully constructed in the orange transformants (Fig. 4e). Hence, scCRISPR/Cpf1 allows for efficient tripleplex genomic integration of in vivo assembled DNA parts in S. cerevisiae.

\section{scCRISPR/Cpf1 for strain building}

To explore its potential for metabolic pathway engineering in $S$. cerevisiae, scCRISPR/Cpf1 was applied to construct and optimize the patchoulol synthesis pathway (Fig. 5a). To introduce the patchoulol synthesis pathway, the rate-limiting step in the MVA pathway was released and the metabolic flux from IPP and DMAPP to FPP through the first round of gene manipulation was enhanced, and the fusion gene of FPPs-PTs, the tHMG1, and the IDI1 were designed to be integrated at 
a
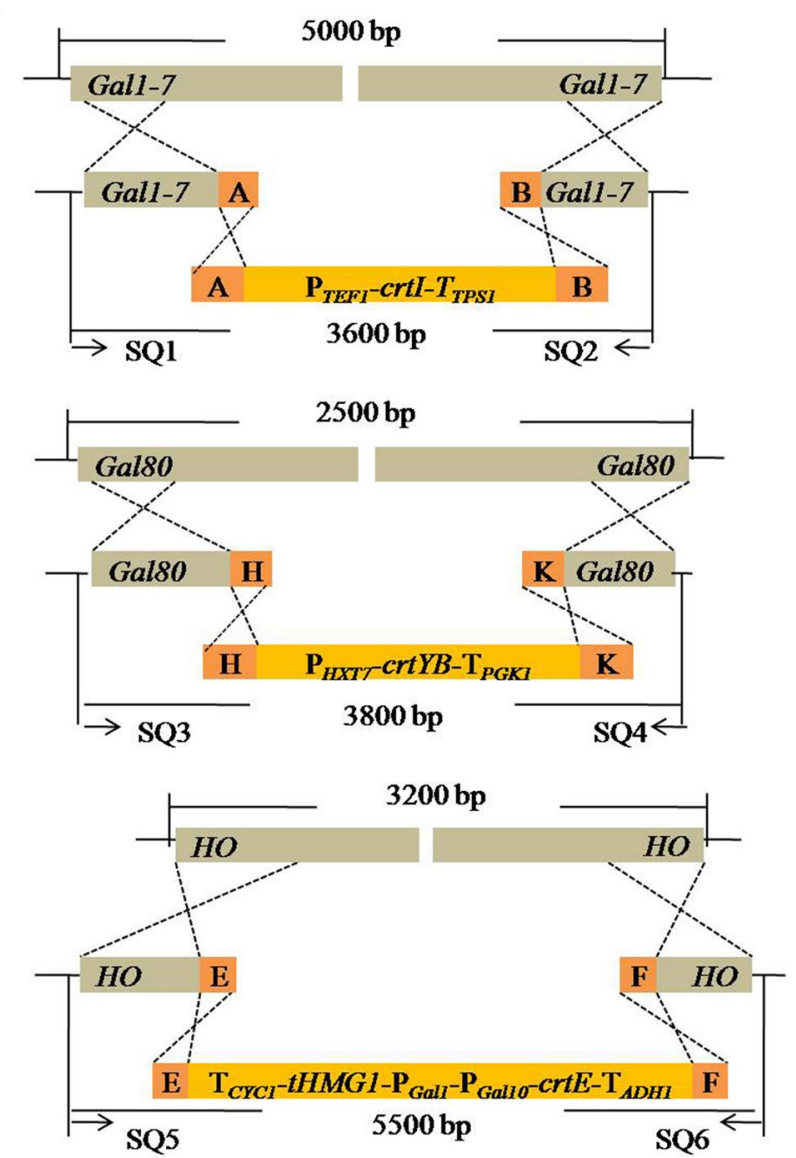

$\mathbf{e}$

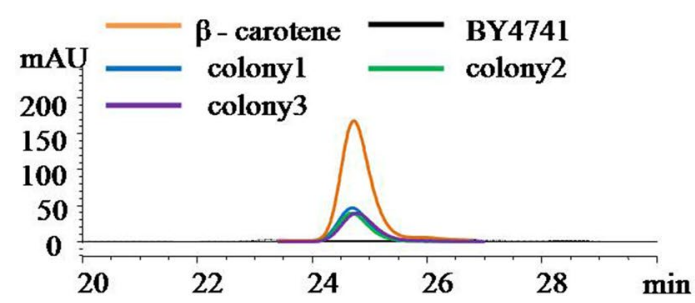

b

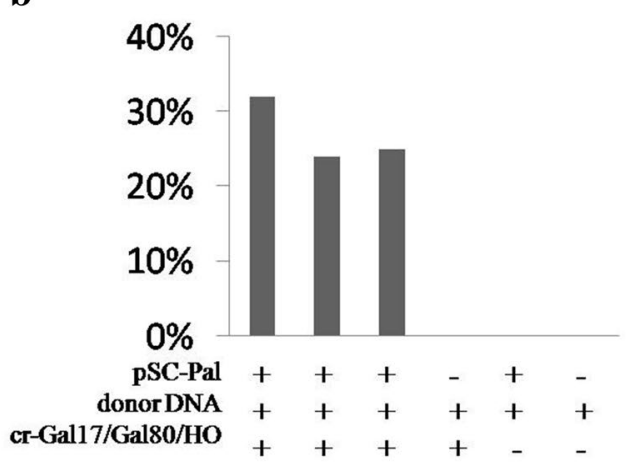

c

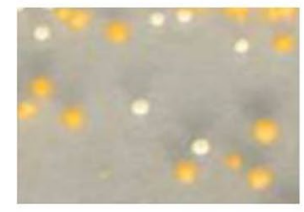

pSC-Pal

donorDNA

cr-Gall 7/Gal80/HO

d
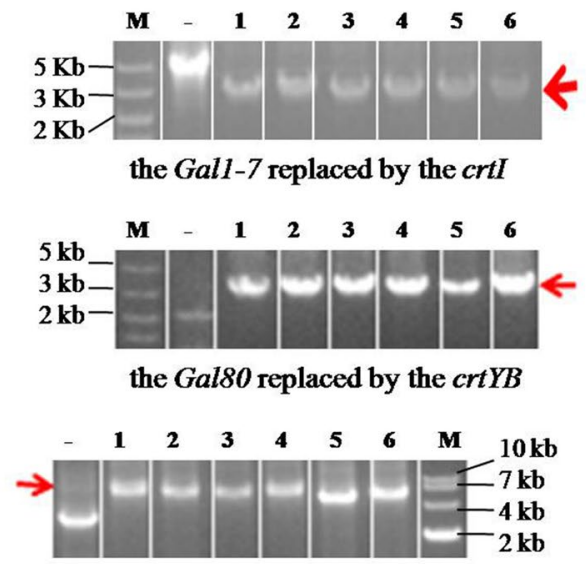

the $H O$ replaced by the $c r t E-t H M G l$

Fig. 4 scCRISPR/Cpf1 facilitated tripleplex genomic integration of in vivo assembled DNA parts in S. cerevisiae. a scCRISPR/Cpf1 facilitated tripleplex genomic integration of $\beta$-carotene synthetic pathway of the crtl, crtYB, and $t H M G 1-c r t E$ into the target sites Gal1-7, Gal80, and HO, respectively. b Efficiency of tripleplex genomic integration assisted by scCRISPR/Cpf1. c Pictures of transformants obtained by co-transforming donor DNA of $\beta$-carotene synthetic pathway with pSC-Pal and cr-Gal17/Gal80/HO. d PCR result of 1 white transformant (-) and 6 orange transformants (1-6) using primer pairs SQ1/SQ2, SQ3/SQ4, and SQ5/SQ6 for integration of crtl at the Gal1-7 (up), crtYB at the Gal80 (middle), and tHMG1-crtE at the HO (bottom), respectively. The horizontal red arrows indicate the PCR bands of correct integration. M: DNA Ladder 5000 or DNA Ladder 10,000. e HPLC analyses of $\beta$-carotene synthesis to evaluate the tripleplex genomic integration. $\beta$-carotene: $\beta$-carotene standard; BY4741: starting yeast strain; Colony1, Colony2, and Colony3: three orange colonies randomly selected. A, B, E, F, H, and K in orange rectangle: 50 bp homologous connector sequences. $P_{T E F 1}, P_{H X T 7}, P_{\text {Gal1, }}$ and $P_{\text {Gal10: }}$ : promoters of the TEF1, HXT7, Gal1, and Gal10, respectively; $T_{T P S 1}, T_{P G K 1}, T_{C Y C 1}$, and $T_{A D H 1}$ : terminators of the TPS1, $P G K 1, C Y C 1$, and $A D H 1$, respectively

the Gal1-7, the Gal80, and the HO locus by scCRISPR/ Cpf1, respectively, generating strain BY-PT-001 (Fig. 5a, Additional file 1: Figs. S1A, S2A, and Table 1). The crRNA plasmid in BY-PT-001 was to be cued by counterselection on a YPD plate that contains 5-fluoroorotic acid, generating strain BY-PT-002 (Table 1). To reduce the metabolic flux from FPP to farnesol and squatene in BY-PT-002 through the second round of gene manipulation, the LPP1 and the DPP1 were designed to be deleted, and the native promoter of the ERG9 $\left(\mathrm{P}_{E R G 9}\right)$ 


\section{$\mathbf{a}$}
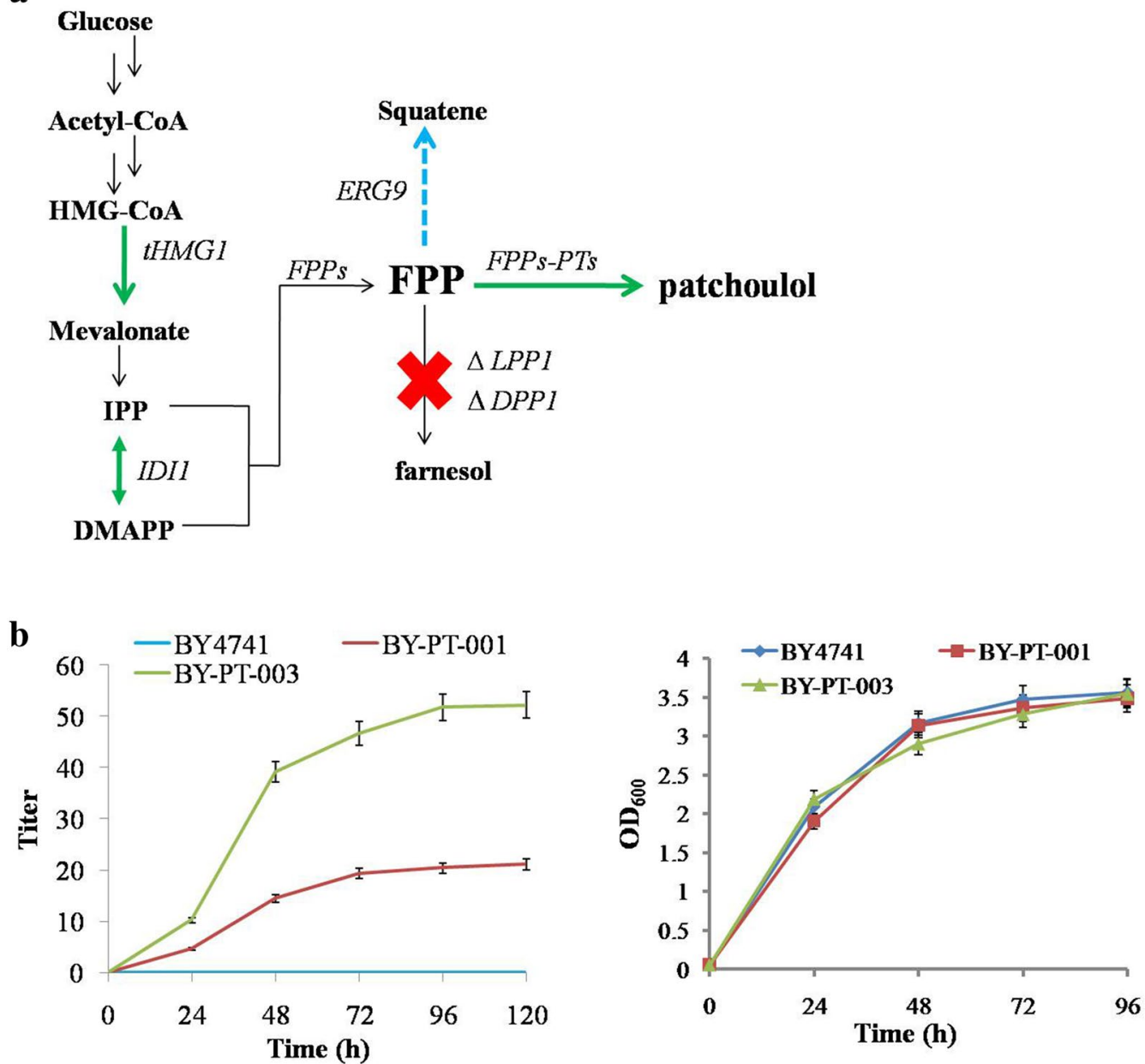

Fig. 5 Application of scCRISPR/Cpf1 in metabolic pathway engineering. a Construction and optimization of the synthesis pathway of patchoulol in S. cerevisiae. Black single arrows represent one-step conversions. Black double arrows represent multiple steps. Green arrows represent the overexpressed genes. Blue arrow represents the downregulated gene by $P_{H X T 1}$ promoter. Red cross represents the deleted gene. HMG-CoA, 3-hydroxy-3-methylglutaryl coenzyme A; IPP, isopentenyl pyrophosphate; DMAPP, dimethylallyl pyrophosphate; IDI1, isopentenyl pyrophosphate isomerase; FPP, farnesyl diphosphate; tHMG1, truncated 3-hydroxy-3-methylglutaryl-coenzyme-A reductase; ERG9, squalene synthase; $L P P 1$ and DPP1, both encoding lipid phosphate phosphatases; FPPs: farnesyl diphosphate synthase; and FPPs-PTs, fusion gene of farnesyl diphosphate synthase and patchoulol synthase. b (Left) The patchoulol titer produced at various time points in strains BY4741, BY-PT-001, and BY-PT-003, respectively. (Right) The growth curves of strains BY4741, BY-PT-001, and BY-PT-003, respectively

was designed to be replaced by a weaker promoter of $\mathrm{P}_{H X T 1}$ by scCRISPR/Cpf1, generating strain BY-PT-003 (Fig. 5a, Additional file 1: Figs. S1B, S4A, and Table 1). We co-transferred the pSC-Pal, the cr-Gal17/Gal80/ $\mathrm{HO}$ array fragment, and the corresponding donor DNA fragments of the FPPs-PTs, the tHMG1, and the IDI1 into yeast cells. The efficiency of correct tripleplex integration is $30 \%(3 / 10)$ in the first round of gene manipulation by scCRISPR/Cpf1 (Additional file 1: Fig. S2). The GC-MS analysis indicated that the yield of patchoulol was $20 \mathrm{mg} / \mathrm{L}$ in the strain BY-PT-001 under shake-flask conditions (Fig. 5b, Additional file 1: Fig. S3). Then, we co-transferred the $\mathrm{pSC}$-Pal, the cr-LPP1/DPP1/ $\mathrm{P}_{E R G 9}$ array fragment, and the corresponding donor DNA fragments of the $\triangle \mathrm{LPP} 1, \triangle \mathrm{DPP} 1$, and the $\mathrm{P}_{H X T 1}$ into cells of BY-PT-002. The efficiency of correct tripleplex integration is $30 \%(3 / 10)$ as well in the second round of gene manipulation by scCRISPR/Cpf1 (Additional file 1: Fig. S4). The GC-MS analysis indicated that the yield of patchoulol was enhanced from $20 \mathrm{mg} / \mathrm{L}$ in the strain BY-PT-001 to $52 \mathrm{mg} / \mathrm{L}$ in the strain BY-PT-003 under shake-flask conditions (Fig. 5b). Hence, scCRISPR/Cpf1 serves as an efficient genome editing tool for metabolic pathway engineering in S. cerevisiae. 
Table 1 The main plasmids and strains used in this study

\begin{tabular}{|c|c|c|}
\hline Plasmids or strains & Characteristics & Source or reference \\
\hline \multicolumn{3}{|l|}{ Plasmid } \\
\hline p416XWP01 & URA3, CENG/ARS4 ori, $A m p^{R}$ & Xie et al. (2013) \\
\hline pMRI-35-crtYB-crtl & $\mathrm{P}_{\text {TEF } 1}-\mathrm{Crtl}-\mathrm{T}_{\text {TPS } 1}, \mathrm{P}_{\mathrm{HXT7}}-\mathrm{CrtYB}-\mathrm{T}_{\text {PGK1}}$ & Xie et al. (2013) \\
\hline pMRI-34-crtE-tHMG1 & $\mathrm{T}_{C Y C 1}-t H M G 1-\mathrm{P}_{\text {Gal1 }}-\mathrm{P}_{\text {Gal10 }}-\mathrm{CrtE}-\mathrm{T}_{A D H 1}$ & Xie et al. (2013) \\
\hline pUMRI-15 & $\mathrm{P}_{\text {TEF } 1} \mathrm{~T}_{\text {TPS } 1}, \mathrm{Kan}^{R}$ & Xie et al. (2013) \\
\hline p426- $P_{\text {SNR52 }}$-gRNA & $P_{S N R 521} A m p^{R}$ & Addgene $(43,803)$ \\
\hline pUMRI-15-Cpf1 & $\mathrm{P}_{\text {TEF } 1}-\mathrm{CPf1}-\mathrm{T}_{\text {TPS } 1}, \mathrm{Kan}^{R}$ & This study \\
\hline p416XWP01-Cpf1 & p416XWP01, $\mathrm{P}_{\text {TEF1 }}-$ Cpf1-T TPS $1, A m p^{R}$ & This study \\
\hline pSC-Pal & 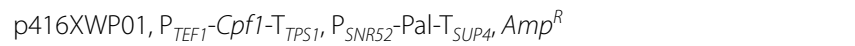 & This study \\
\hline pUMRI-15-MEL1 & pUMRI-15, $\mathrm{P}_{\text {TEF }}-$ MEL 1-T TPS1, $_{\text {Tan }}{ }^{R}$ & This study \\
\hline pUMRI-15-FPPs-PTS & pUMRI-15, $\mathrm{P}_{\text {TEF1 }}-$ FPPS $-P T_{S-T} T_{\text {TPS } 1}, \mathrm{Kan}^{R}$ & This study \\
\hline p416XWP01-IDI1 & p416XWP01, $\mathrm{P}_{\text {Gallo- }}-\mid \mathrm{D} / 1-\mathrm{T}_{A D H 1}, A m p^{R}$ & This study \\
\hline \multicolumn{3}{|l|}{ Strain } \\
\hline BY4741 & MATa, his $3 \Delta 1$, leu2 $\Delta 0$, met $15 \Delta 0$, ura3 $\Delta 0$ & Brachmann et al. (1998) \\
\hline BY-PT-001 & 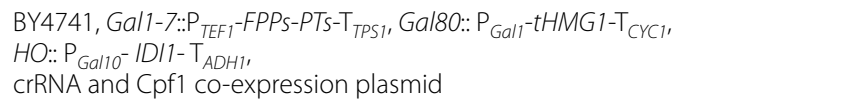 & This study \\
\hline BY-PT-002 & 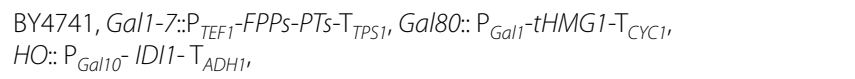 & This study \\
\hline BY-PT-003 & BY-PT-002, $\triangle L P P 1, \triangle D P P 1, \mathrm{P}_{E R G 9: P}: \mathrm{P}_{H X T 1}, \mathrm{CrRNA}$ and $\mathrm{Cpf1}$ co-expression plasmid & This study \\
\hline
\end{tabular}

\section{Discussion}

Nowadays, the advancement in the CRISPR/Cas systems is revolutionizing the biology, medicine, and biotechnology fields. Cpf1, derived from a class $2 /$ type V CRISPR system, is a Cas effector protein with unique features, which may enable CRISPR/Cpf1 to be an alternative to CRISPR/Cas9 in genome editing. The CasEMBLR is the most efficient method reported so far for CRISPR/Cas9 facilitated genome editing of $S$. cerevisiae. The efficiencies of singleplex, doubleplex, and tripleplex genome editing facilitated by the CasEMBLR are 97, 58, and $30.6 \%$, respectively (Jakočiūnas et al. 2015). In this study, the scCRISPR/Cpf1 enables singleplex and tripleplex genomic integration of in vivo assembled DNA parts with efficiencies of 80 and 32\%, respectively. Thus, the CRISPR/Cpf1-based scCRISPR/Cpf1 is comparable to the CRISPR/Cas9-based CasEMBLR.

In the singleplex and tripleplex genome editing, the deleted DNA length of the Gal1-7, the Gal80, and the $H O$ are $5,2.5$, and $3.2 \mathrm{~kb}$, respectively (Figs. 3a, 4a); the desired DNA length for integration into the Gal1-7 locus, the Gal8O locus, and the $H O$ locus are 3.6, 3.8, and $5.5 \mathrm{~kb}$, respectively (Fig. 4a). Our results showed that the length of targeted fragment within $5.5 \mathrm{~kb}$ would not significantly affect the efficiency of gene editing, the reason for which might be that the HR is robust in S. cerevisiae.

Ribonuclease activity that functions in crRNA processing is one of the most important features of Cpf1, which simplifies the construction of multiplex-crRNA cassette with a crRNA array. The plasmid HR is efficient in S. cerevisiae. Our results demonstrated that the characteristics of complementary palindromic sequence for self-cleaving and plasmid HR in S. cerevisiae are critical parameters for scCRISPR/Cpf1 working. The HPLC analysis of $\beta$-carotene was just used to assess the efficiency of multiplex genomic integration facilitated by scCRISPR/ Cpf1, so that the quantitative analysis of $\beta$-carotene does not need to be performed. Although the patchoulol yield under shake-flask conditions in strain BY-PT-003 engineered in this study is the highest production reported so far, the biomass of the strains was too low. The strain engineering and pathway optimization in this study were just used to assess the applicability of scCRISPR/Cpf1 in desired metabolic pathway engineering in S. cerevisiae, so that the patchoulol-producing strain was not further optimized to enhance the yield of patchoulol.

\section{Conclusion}

In this study, we developed a scCRISPR/Cpf1 method that allows for genomic integration by circumventing any cloning steps without compromising efficiency, which provides an alternative to CRISPR/Cas9 in metabolic pathway engineering in $S$. cerevisiae. To sum up,the scCRISPR/Cpf1 method shown in this study enriches the current set of tools available for strain engineering in $S$. cerevisiae. 


\section{Methods}

\section{Strains, media and reagents}

Saccharomyces cerevisiae BY4741 (MATa, his3 1 , leu2 $\Delta 0$, met15 $\Delta 0$, ura3 $\Delta 0$ ) was used as the parent strain (Brachmann et al. 1998). The $\alpha$-galactosidase encoding gene (MEL1) was amplified from the $S$. cerevisiae Y187. All engineered yeast strains are listed in Table 1 . Medium of $1 \%$ yeast extract, $2 \%$ peptone, and $2 \%$ D-glucose (YPD) was used for yeast cultivation. Uracil auxotrophy synthetic medium (SD-URA) and leucine auxotrophy synthetic medium (SD-LEU) purchased from FunGenome (FunGenome, China), adding X- $\alpha$-gal $(40 \mathrm{ng} / \mathrm{mL})$ or geneticin G418 when necessary, were used for selection and cultivation of the recombinants. Yeast cells were propagated at $30{ }^{\circ} \mathrm{C}$. Escherichia coli DH5 $\alpha$ (Takara, Japan) was used for transformation and plasmid amplification and extraction. KOD-FX (TOYOBO, Japan) was used for following the manufacturer's recommendations for all the PCRs. X- $\alpha$-gal and the standard $\beta$-carotene and patchoulol were purchased from Sigma (Sigma-Aldrich, USA). Restriction endonucleases and T4 DNA ligase were purchased from Thermo Scientific.

\section{Construction of plasmid and donor DNA}

To create a set of self-cleaving complementary palindromic crRNA and Cpf1 co-expression plasmids, the codon-optimized Cpf1 from Francisella novivida was first ligated into pUMRI-15 using BamHI and NheI, generating pUMRI-15-Cpf1. Then, the Cpf1 cassette was amplified from pUMRI-15-Cpf1 using primer pair P1/ $\mathrm{P} 2$, and ligated into the BssHII linearized p416XWP01 using One Step Cloning Kit (Vazyme, China), generating $\mathrm{p} 416 \mathrm{XWP} 01-\mathrm{Cpf1}$. The complementary palindromic crRNA sequence starting with "TTTG" is included in the primer P4. The fragments amplified from the $\mathrm{p} 426$ $\mathrm{P}_{S N R 52^{-}}$gRNA using primer pairs $\mathrm{P} 3 / \mathrm{P} 4$ were ligated into the p416XWP01-Cpf1 using $M l u \mathrm{I}$ and KpnI, generating pSC-Pal. To create the non-palindromic crRNA and Cpf1 co-expression plasmid pCon-NP, the fragment amplified from the $\mathrm{p}_{426}-\mathrm{P}_{\text {SNR52 }}$-gRNA using primer pair P3/ NP-R was ligated into the p416XWP01-Cpf1. To create the non-PAM crRNA and Cpf1 co-expression plasmid pCon-MP, the fragment amplified from the $\mathrm{p}^{426-\mathrm{P}_{\text {SNR52- }}}$ gRNA using primer pair P3/MP-R was ligated into the p416XWP01-Cpf1.

The KanMX fragment was amplified from pUMRI-15 with primers KM-F/KM-R.

Donor DNA for gene over-expression of each locus contained a gene expression cassette and two homology flanking fragments (upstream and downstream). The donor DNA expression cassette and homology flanking fragments that contained $50 \mathrm{bp}$ connector sequences allow for in vivo assembly. To create pUMRI-15-MEL1,
MEL1 gene was amplified from S. cerevisiae Y187 with primer pair MEL-F/MEL-R, and ligated into pUMRI-15 using BamHI and NheI. To create pUMRI-15-FPPs-PTs, the codon-optimized patchoulol synthase $(P T s)$ from pachouli (Pogostemon cablin) synthesized in Ruimian (Ruimian, China) was first fused with farnesyl diphosphate synthase (FPPs) using primer pairs P7/P8 and P9/ P10, and ligated into pUMRI-15 using BamHI and XhoI. To create p416XWP01-IDI1, the isopentenyl pyrophosphate isomerase (IDI1) amplified from the genome of BY4741 using primer pair P11/P12 was ligated into p416XWP01 using EcoRI and NotI. The donor DNA expression cassettes of MEL1, crtI, FPPs-PTs, crtYB, tHMG1-crtE, $t H M G 1$, and IDI1 were amplified from pUMRI-15-MEL1, pMRI-35-crtYB-crtI, pUMRI-15FPPs-PTs, pMRI-34-crtE-tHMG1, and p416XWP01IDI1 with primer pairs P13/P14, P15/P16, P17/P18, P19/ $\mathrm{P} 20$, and $\mathrm{P} 21 / \mathrm{P} 18$, respectively. The homology flanking fragments for integration of the $M E L 1$ or the $c r t I$ or the FPPs-PTs into the Gal1-7 were amplified from the genome of BY4741 using primer pairs P22/P23 and P24/ P25. The homology flanking fragments for integration of the $\operatorname{crt} Y B$, the $t H M G 1-c r t E$, the $t H M G 1$, and the IDI1 were amplified from the genome of BY4741using primer pairs P26/P27 and P28/P29, P30/P31 and P32/P33, P26/ P34 and P35/P29, and P30/P36 and P32/P33, respectively.

Donor DNA for gene knockout of each locus that contained two homology fragments (upstream and downstream), which contained $50 \mathrm{bp}$ connector sequences allow for in vivo assembly. The homology fragments for knockout of LPP1 and DPP1 were amplified from the genome of BY4741 using primer pairs P37/P38 and P39/ P40, P41/P42 and P43/P44, respectively. Donor DNA for replacement of $\mathrm{P}_{E R G 9}$ by $\mathrm{P}_{H X T 1}$ was amplified from the genome of BY4741 using primer pairs $\mathrm{P} 45 / \mathrm{P} 46, \mathrm{P} 47 / \mathrm{P} 48$, and $\mathrm{P} 49 / \mathrm{P} 50$. All primers used in this study are listed in Additional file 1: Table S1. All expression cassettes and homology sequences of donor DNA are shown in Additional file 1: Table S2.

\section{Site-specific crRNA homology fragment}

The sequences of crRNAs randomly selected with PAM of $5^{\prime}$-TTTN- $3^{\prime}$ are listed in Additional file 1: Table S1. The $90 \mathrm{bp}$ of upstream homologous arm of the small DNA fragment is composed of $71 \mathrm{bp}$ of promoter $\mathrm{P}_{S N R 52}$ and the first $19 \mathrm{bp}$ of direct repeat. The $90 \mathrm{bp}$ of downstream homologous arm of the small DNA fragment is composed of $20 \mathrm{bp}$ of $\mathrm{T}_{\text {SUP4 }}$, and $70 \mathrm{bp}$ of homology fragments with plasmid. To prepare the $203 \mathrm{bp}$ of DNA fragment that contains singleplex site-specific crRNA and the $287 \mathrm{bp}$ of DNA fragment that contains tripleplex site-specific crRNA, the PCR is performed as described previously (Arbab et al. 2015). The singleplex crRNA 
targeting to the $A D E$ (cr-ADE2), the singleplex crRNA targeting to the Gal1-7 (cr-Gal17), the tripleplex crRNA array targeting to the Gal1-7, Gal80, and $H O$, and the tripleplex crRNA array targeting to the $L P P 1, D P P 1$, and $\mathrm{P}_{E R G 9}$ were prepared with primer pairs Up-F/P5, Up-F/ P6, Up-F/cr17-80-HO-R1/cr17-80-HO-R2, and Up-F/ crL-D-9-R1/crL-D-9-R2, respectively. The primers synthesized in Ruimian (Ruimian, China) are listed in Additional file 1: Table S1.

\section{Yeast transformation}

Saccharomyces cerevisiae BY4741 was used as the starting strain. Electroporation was used for the yeast transformation (Kawai et al. 2010).

To test the functionality of scCRISPR/Cpf1 for selfcleaving, pCon-NP, pCon-MP, and pSC-Pal were transformed to yeast cells, respectively, and plated on SD-URA.

To test whether plasmid HR of yeast functions to repair self-cleaved linearized pSC-Pal in S. cerevisiae, the $\operatorname{KanMX}$ fragment with pCon-NP, and the KanMX fragment with pSC-Pal were transformed to yeast cells, respectively, and plated on SD-URA containing the geneticin G418.

To test whether plasmid $\mathrm{HR}$ of yeast functions to repair self-cleaved linearized pSC-Pal with a small site-specific crRNA fragment, cr-ADE2 and pLEU-Pal were co-transformed into yeast cells, and plated on SD-LEU.

To test the efficiency of targeted singleplex genomic integration facilitated by scCRISPR/Cpf1 in S. cerevisiae, pSC-Pal were co-transformed, respectively, with donor DNA of MEL1 and the small DNA fragment that contains the crRNA targeting to the Gal1-7. Transformation of the donor DNA of MEL1, the small DNA fragment that contains the crRNA targeting to the Gal1-7, and pCon-NS,respectively, was used as three individual controls. The transformants were selected in SD-URA plates containing X- $\alpha$-gal. To test the efficiency of tripleplex genomic integration of in vivo assembled DNA parts facilitated by scCRISPR/Cpf1, pSC-Pal was cotransformed with the crRNA-Gal1-7/Gal80/HO array fragment and the corresponding donor DNA of the $\beta$-carotene synthetic pathway into yeast cells.

To build the strain BY-PT-001, we co-transferred the crRNA-Gal1-7/Gal80/HO array fragment, the corresponding donor DNA fragments of the FPPs-PTs, the $t H M G 1$, and the IDI1, and the pSC-Pal into yeast cells. To obtain strain BY-PT-002, crRNA plasmid in BY-PT-001 was counterselected on a YPD plate that contains 5-fluoroorotic acid. To build the strain BY-PT-003, we co-transformed the crRNA-LPP1/DPP1/P $\mathrm{P}_{E R G 9}$ array fragment, the corresponding donor DNA fragments of $\triangle \mathrm{LPP} 1$, $\triangle \mathrm{DPP} 1, \mathrm{P}_{H X T 1}$, and pSC-Pal into cells of BY-PT-002.

\section{Strain genotyping}

For genotyping of colonies with corresponding phenotype, 6 colonies randomly selected with corresponding phenotype and 1 colony without phenotype as control were analyzed by colony PCR. For genotyping of colonies without obvious phenotype, 10 colonies randomly selected were analyzed by colony PCR. Each of the primer pair was designed as: one that is complementary to the flanking sequence 50-bp upstream of the upstream homology sequence, one that is complementary to the flanking sequence 50-bp downstream of the downstream homology sequence. Primer pairs SQ1/ SQ2, SQ3/SQ4, SQ5/SQ6, SQ7/SQ8, SQ9/SQ10, and SQ11/SQ12 were used to identify correct integration of the Gal1-7, the Gal80, the HO, the LPP1, the DPP1, and the $\mathrm{P}_{E R G 9}$, respectively.

\section{Strain cultivation and analysis of $\beta$-carotene and patchoulol}

The strains for product analysis were pre-cultured in $5 \mathrm{~mL} \mathrm{YPD}$ at $30^{\circ} \mathrm{C}$ and $220 \mathrm{rpm}$ for $24 \mathrm{~h}$. Pre-cultures were inoculated to an initial $\mathrm{OD}_{600}$ of 0.05 in $50 \mathrm{~mL}$ YPD in $250 \mathrm{~mL}$ flasks and grown in the same conditions for $72 \mathrm{~h}$. $\beta$-carotene was extracted from fermentation products using the hot $\mathrm{HCl}$-acetone method (Xie et al. 2013). An Agilent 1000 system equipped with an Agilent C18 column $(5 \mu \mathrm{m}, 4.6 \mathrm{~mm} \times 250 \mathrm{~mm})$ was used for HPLC analysis. The mobile phase consisting of acetonitrile: methanol: isopropanol $=5: 3: 2$ with a flow rate of $1 \mathrm{~mL} / \mathrm{min}$ at $40{ }^{\circ} \mathrm{C}$, and the UV/VIS signals were detected at a wavelength of $450 \mathrm{~nm}$.

Patchoulol produced during fermentation was extracted using the dodecane method (Albertsen et al. 2011). A GC-MS-QP2010 ultra system, which was equipped with a DB-5 capillary column (30 $\mathrm{m} \times 0.25 \mathrm{~mm}$ i.d., $0.25 \mu \mathrm{m}$ film thickness) and using helium as carrier gas at a flow rate of $1.2 \mathrm{~mL} / \mathrm{min}$, was used for the identification and quantitative analysis of patchoulol. A split/splitless injector was used in the splitless mode. The initial oven temperature was $80{ }^{\circ} \mathrm{C}$, and injector temperature was $250{ }^{\circ} \mathrm{C}$. The oven temperature was increased to $120{ }^{\circ} \mathrm{C}$ after $1 \mathrm{~min}$ at a rate of $108{ }^{\circ} \mathrm{C} / \mathrm{min}$ and subsequently increased to $160^{\circ} \mathrm{C}$ at a rate of $3{ }^{\circ} \mathrm{C} / \mathrm{min}$. The oven temperature was finally increased to $270{ }^{\circ} \mathrm{C}$ at a rate of $10^{\circ} \mathrm{C} / \mathrm{min}$ and held for $5 \mathrm{~min}$ at this temperature.

\section{Additional file}

Additional file 1: Figure S1. Graphical depiction of the positions of the genomic target sites of Gal1-7, Gal80, HO, LPP1, DPP1, and PERG9. Figure S2. scCRISPR/Cpf1-facilitated construction of the patchoulol synthesis pathway in S. cerevisiae. A: scCRISPR/Cpf1-facilitated genomic integration of the patchoulol synthesis pathway in the first round. $A, B, C, D, G$, and $F$ 
in orange rectangle: 50 bp homologous connector sequences. $P_{\text {TEF }}, P_{\text {Gall, }}$ and $P_{\text {Gallo: }}$ : promoters of the TEF1, Gal1, and Gal10, respectively; $T_{T P S 1}, T_{C Y C 1}$, and $T_{A D H}$ : terminators of the TPS1, $C Y C 1$, and $A D H 1$, respectively. $B: P C R$ result of $B Y 4741$ (-) and ten randomly selected transformants, line \#1-10, using primer pairs SQ1/SQ2, SQ3/SQ4, and SQ5/SQ6 for integration of FPPs-PTs at the Gal1-7 (Up), tHMGI at the Gal8O (Middle), and IDI at the HO (Bottom), respectively, in the first round. The expected sizes of the correct integration at Gal 1-7, Gal80, and HO are 4500 bp, 3200 bp, and 2500 bp, instead of the native $5000 \mathrm{bp}, 2500 \mathrm{bp}$, and $3200 \mathrm{bp}$, respectively. M: DNA Ladder 5000. Figure S3. Identification of patchoulol produced in the strain BY-PT-001. A: GC analysis of patchoulol strandard (left) and product in the strain BY-PT-001 (right). B: GC-MS analysis of patchoulol strandard (up) and product in the strain (bottom). Figure S4. scCRIPR/ Cpf1-facilitated optimization of the patchoulol synthesis pathway in $S$. cerevisiae. A: scCRISPR/Cpf1 facilitated optimization of the patchoulol synthesis pathway in the second round. B: PCR result of BY4741 (-) and ten randomly selected transformants, line \#1-10, using primer pairs SQ7/SQ8 (Up), SQ9/SQ10 (Middle), and SQ11/SQ12 (Bottom) for deletion of LPP1 and $L P P 1$, and replacement of $P_{E R G 9}$ by $P_{H X T 1}$, respectively, in the second round. The horizontal red arrows indicate the correct integration. J, L, M, and $\mathrm{N}$ in orange rectangle: $50 \mathrm{bp}$ homologous connector sequences. The expected sizes of the correct integration at the $\angle P P 1, D P P 1$, and $P_{E R G 9}$ are $700 \mathrm{bp}, 700 \mathrm{bp}$, and $1500 \mathrm{bp}$, instead of the native $2000 \mathrm{bp}, 1500 \mathrm{bp}$, and $750 \mathrm{bp}$, respectively. M: DNA Ladder 5000. Table S1: Primers and oligonucleotides used in this study. crRNA sequences are indicated in bold, and direct repeat sequences are indicated in underline. Table 2: Donor DNA flank and expression cassette sequences. Connector sequences are indicated in bold.

\section{Abbreviations}

HR: homologous recombination; DSB: double-stranded break; CRISPR: clustered regularly interspaced short palindromic repeats; PAM: Protospacer Adjacent Motif; PCR: polymerase chain reaction.

\section{Authors' contributions}

ZHL and FQW designed experiments. ZHL performed the experiments. FQW and DZW contributed reagents and materials. ZHL and FQW drafted the manuscript. All authors read and approved the final manuscript.

\section{Acknowledgements}

The authors sincerely thank Prof. Hongwei Yu (Zhejiang University) for providing plasmids of p416XWP01, pMRI-34-crtE-tHMG1, pMRI-35-crtYB-crtl, pUMRI15 , and strain of S. cerevisiae BY4741.

\section{Competing interests}

The authors declare that they have no competing interests.

\section{Availability of data materials}

The datasets supporting the conclusions of this article are included in the main manuscript.

\section{Consent for publication}

The authors approved the consent for publishing the manuscript.

\section{Ethics approval and concept to participate}

All the authors have read and agreed the ethics for publishing the manuscript.

\section{Funding}

This research was financially supported by the National Natural Science Foundation of China (No. 21776075)

\section{Publisher's Note}

Springer Nature remains neutral with regard to jurisdictional claims in published maps and institutional affliations.

Received: 21 June 2018 Accepted: 21 July 2018

Published online: 30 July 2018

\section{References}

Albertsen L, Chen Y, Bach LS, Rattleff S, Maury J, Brix S (2011) Diversion of flux toward sesquiterpene production in Saccharomyces cerevisiae by fusion of host and heterologous enzymes. Appl Environ Microbiol 77:1033-1040

Arbab M, Srinivasan S, Hashimoto T, Geijsen N, Sherwood RI (2015) Cloningfree CRISPR. Stem Cell Rep 5:908-917

Brachmann CB, Davies A, Cost GJ, Caputo E, Li J, Hieter P, Boeke JD (1998) Designer deletion strains derived from Saccharomyces cerevisiae S288C: a useful set of strains and plasmids for PCR-mediated gene disruption and other applications. Yeast 14:115-132

Carbonell P, Currin A, Jervis AJ, Rattray NJ, Swainston N, Yan C (2016) Bioinformatics for the synthetic biology of natural products: integrating across the design-build-test cycle. Nat Prod Rep 33:925

Dai Z, Wang B, Liu Y, Shi M, Wang D, Zhang XL (2014) Producing aglycons of ginsenosides in bakers'yeast. Sci Rep 4:3698

Dicarlo JE, Norville JE, Mali P, Rios X, Aach J, Church GM (2013) Genome engineering in Saccharomyces cerevisiae using CRISPR-Cas systems. Nucleic Acids Res 41:4336-4343

Engels B, Dahm P, Jennewein S (2008) Metabolic engineering of taxadiene biosynthesis in yeast as a first step towards Taxol (Paclitaxel) production. Metab Eng 10:201-206

Horwitz A, Walter J, Schubert M, Kung S, Hawkins K, Platt D (2015) Efficient multiplexed integration of synergistic alleles and metabolic pathways in yeasts via CRISPR-Cas. Cell Syst 1:88-96

Hwang WY, Fu Y, Reyon D, Maeder ML, Tsai SQ, Sander JD (2013) Efficient genome editing in zebrafish using a CRISPR-Cas system. Nat Biotechnol 31:227-229

Jakočiūnas T, Rajkumar AS, Zhang J, Arsovska D, Rodriguez A, Jendresen CB (2015) CasEMBLR: Cas9-facilitated multiloci genomic integration of in vivo assembled DNA parts in Saccharomyces cerevisiae. Acs Synth Biol 4:1226

Kawai S, Hashimoto W, Murata K (2010) Transformation of Saccharomyces cerevisiae and other fungi. Bioeng Bugs 1:395-403

Kondo T, Tezuka H, Ishii J, Matsuda F, Ogino C, Kondo A (2012) Genetic engineering to enhance the Ehrlich pathway and alter carbon flux for increased isobutanol production from glucose by Saccharomyces cerevisiae. J Biotechnol 159:32-37

Li H, Shen Y, Wu M, Hou J, Jiao C, Li Z (2016) Engineering a wild-type diploid Saccharomyces cerevisiae strain for second-generation bioethanol production. Bioresour Bioprocess 3:51

Marienhagen J, Bott M (2013) Metabolic engineering of microorganisms for the synthesis of plant natural products. J Biotechnol 163:166-178

Meadows AL, Hawkins KM, Tsegaye Y, Antipov E, Kim Y, Raetz L (2016) Rewriting yeast central carbon metabolism for industrial isoprenoid production. Nature 537:694

Nielsen J, Keasling JD (2016) Engineering cellular metabolism. Cell 164:1185-1197

Paddon CJ, Keasling JD (2014) Semi-synthetic artemisinin: a model for the use of synthetic biology in pharmaceutical development. Nat Rev Microbiol 12:355

Ro DK, Paradise EM, Ouellet M, Fisher KJ, Newman KL, Ndungu JM, Ho KA, Eachus RA, Ham TS, Kirby J, Chang MC, Withers ST, Shiba Y, Sarpong R, Keasling JD (2006) Production of the antimalarial drug precursor artemisinic acid in engineered yeast. Nature 440:940

Sander JD, Joung JK (2014) CRISPR-Cas systems for editing, regulating and targeting genomes. Nat Biotechnol 32:347-355

Schwartz C, Shabbirhussain M, Frogue K, Blenner M, Wheeldon I (2017) Standardized markerless gene integration for pathway engineering in Yarrowia lipolytica Acs. Synth Biol 6:402

Siddiqui MS, Choksi A, Smolke CD (2015) A system for multi-locus chromosomal integration and transformation-free selection marker rescue. Fems Yeast Res 14:1171-1185

Smanski MJ, Bhatia S, Zhao D, Park Y, Baw L, Giannoukos G (2014) Functional optimization of gene clusters by combinatorial design and assembly. Nat Biotechnol 32:1241-1249

Storici F, Lewis LK, Resnick MA (2001) In vivo site-directed mutagenesis using oligonucleotides. Nat Biotechnol 19:773-776

Storici F, Durham CL, Gordenin DA, Resnick MA (2003) Chromosomal sitespecific double-strand breaks are efficiently targeted for repair by oligonucleotides in yeast. Proc Natl Acad Sci USA 100:14994-14999 
Swiat MA, Dashko S, Den RM, Wijsman M, Van DOJ, Daran JM, Daran-Lapujade P (2017) FnCpf1: a novel and efficient genome editing tool for Saccharomyces cerevisiae. Nucleic Acids Res 45:12585-12598

Verwaal R, Buiting-Wiessenhaan N, Dalhuijsen S, Roubos JA (2018) CRISPR/ Cpf1 enables fast and simple genome editing of Saccharomyces cerevisiae. Yeast 35:201-211. https://doi.org/10.1002/yea.3278

Wang S, Sheng D, Wang P, Yong T, Yi W (2017) Genome editing in Clostridium saccharoperbutylacetonicum N1-4 with the CRISPR-Cas9. Syst Appl Environ Microbiol 83:AEM.00233

Westbrook AW, Moo-Young M, Chou CP (2016) Development of a CRISPRCas9 tool kit for comprehensive engineering of Bacillus subtilis. Appl Environ Microbiol 82:4876

Xie W, Liu M, Lv X, Lu W, Gu J, Yu H (2013) Construction of a controllable $\beta$-carotene biosynthetic pathway by decentralized assembly strategy in Saccharomyces cerevisiae. Biotechnol Bioeng 111:125-133
Xie W, Ye L, Lv X, Xu H, Yu H (2015) Sequential control of biosynthetic pathways for balanced utilization of metabolic intermediates in Saccharomyces cerevisiae. Metab Eng 28:8

Yao X, Wang X, Hu X, Liu Z, Liu J, Zhou H (2017) Homology-mediated end joining-based targeted integration using CRISPR/Cas9. Cell Res 27:801

Yu H, Zhu B, Zhan Y (2017) Microbial transformation of artemisinin by Aspergillus terreus. Bioresour Bioprocess 4:33

Zetsche B, Heidenreich M, Mohanraju P, Fedorova I, Kneppers J, Degennaro EM (2017) Multiplex gene editing by CRISPR-Cpf1 using a single crRNA array. Nat Biotechnol 35:31

Zhou J, Wu R, Xue X, Qin Z (2016) CasHRA (Cas9-facilitated homologous recombination assembly) method of constructing megabase-sized DNA. Nucleic Acids Res 44:e124

\section{Submit your manuscript to a SpringerOpen ${ }^{\circ}$ journal and benefit from:}

- Convenient online submission

- Rigorous peer review

- Open access: articles freely available online

- High visibility within the field

- Retaining the copyright to your article

Submit your next manuscript at $\boldsymbol{\nabla}$ springeropen.com 